Review Article

\title{
Molecular and Cellular Pathways Contributing to Joint Damage in Rheumatoid Arthritis
}

\author{
Qinghua Fang, Chun Zhou, and Kutty Selva Nandakumar 1 \\ SMU-KI United Medical Inflammation Center, School of Pharmaceutical Sciences, Southern Medical University, Guangzhou, China
}

Correspondence should be addressed to Kutty Selva Nandakumar; nandakumar@smu.edu.cn

Received 12 January 2020; Accepted 24 February 2020; Published 17 March 2020

Academic Editor: Ronald Gladue

Copyright (C) 2020 Qinghua Fang et al. This is an open access article distributed under the Creative Commons Attribution License, which permits unrestricted use, distribution, and reproduction in any medium, provided the original work is properly cited.

\begin{abstract}
Rheumatoid arthritis is a chronic autoimmune syndrome associated with several genetic, epigenetic, and environmental factors affecting the articular joints contributing to cartilage and bone damage. Although etiology of this disease is not clear, several immune pathways, involving immune ( $\mathrm{T}$ cells, $\mathrm{B}$ cells, dendritic cells, macrophages, and neutrophils) and nonimmune (fibroblasts and chondrocytes) cells, participate in the secretion of many proinflammatory cytokines, chemokines, proteases (MMPs, ADAMTS), and other matrix lysing enzymes that could disturb the immune balance leading to cartilage and bone damage. The presence of autoantibodies preceding the clinical onset of arthritis and the induction of bone erosion early in the disease course clearly suggest that initiation events damaging the cartilage and bone start very early during the autoimmune phase of the arthritis development. During this process, several signaling molecules (RANKL-RANK, NF- $\kappa$ B, MAPK, NFATc1, and Src kinase) are activated in the osteoclasts, cells responsible for bone resorption. Hence, comprehensive knowledge on pathogenesis is a prerequisite for prevention and development of targeted clinical treatment for RA patients that can restore the immune balance improving clinical therapy.
\end{abstract}

\section{Introduction}

Rheumatoid arthritis (RA) is a chronic, inflammatory syndrome comprised of various disease phenotypes. RA is characterized by aggressive synovial hyperplasia causing destruction of articular joints. A combination of genetic, epigenetic, and environmental factors is responsible for the onset and development of RA. An array of susceptible genes (human leukocyte antigen (HLA) class II and more than 100 susceptibility loci including PTPN22, PADI4, TRAF1, and CTLA4), nongenetic factors (sex hormones, smoking, periodontal infection, and microbiota), immune (macrophages, dendritic cells, mast cells, neutrophils, T cells, and B cells) and nonimmune (fibroblasts and chondrocytes) cells, and inflammatory mediators (autoantibodies, cytokines, chemokines, and proteases) are collectively involved in the inflammatory processes targeting the cartilage and bone effectuating functional loss of joints (Figure 1) [1].

The synovium is one of the major target tissues in RA [2]. During joint inflammation, macrophage-like synoviocytes
(MLS) and fibroblast-like synoviocytes (FLS) proliferate to form the pannus, which invades and destroys the cartilage. These cells are the major sources of factors that can promote inflammation and joint destruction. Autoantibodies contribute to the inflammatory process by acting as the mediator of joint inflammation and bone erosion [3]. $50-80 \%$ of RA patients have autoantibodies depending on the duration of the disease. Autoantibodies can initiate inflammatory effector pathways, which affect chondrocytes and the cartilage causing release of extracellular matrix (ECM) components. In this context, glycosylation of autoantibodies is crucial. Decreased IgG-Fc sialylation is associated with RA and osteoclastogenesis, while an increase in sialylation decreased inflammatory bone loss [4]. Bone erosion and loss of physical function in arthritis begin early and progress along with disease severity. The main triggers of bone erosion are an inflamed synovium, proinflammatory cytokines, autoantibodies, and receptor activator of nuclear factor $\kappa \mathrm{B}$ ligand (RANKL). Breakdown of self-tolerance causes activation of immune and nonimmune cells resulting in the production 




Figure 1: Different phases in RA pathogenesis. (1) Genetic, epigenetic, and environmental factors contribute to arthritis progression. Multiple environmental risk factors (for example, smoking, pollutants, or microbes), when come in contact with the mucosal sites, are most likely responsible for causing local inflammatory events and immune system activation inducing epigenetic modifications and protein posttranslational modifications (PTMs) [59], before crossing the threshold to trigger disease in genetically vulnerable people. (2) Dendritic cells presenting altered self or related peptides to $\mathrm{T}$ cells (breakdown of tolerance mechanisms) leads to the activation of $\mathrm{T}$ and $\mathrm{B}$ cells effectuating synthesis of cytokines and autoantibodies. Progressively, these autoantibodies are produced more and more, which recognize several neoepitopes by the process of epitope spreading, and gets overt during the onset of the clinical disease [1]. (3) Disease development involves autoimmune responses against both posttranslationally modified and unmodified self-antigens, which starts many years before the subclinical synovitis and appearance of clinical symptoms [60]. (4) Autoantibodies induced during this preclinical phase can also be responsible for bone erosion and pain. Before the onset of inflammation, these alterations could reduce overall functions of the joints. After the autoantibodies start binding to different epitopes and form immune complexes, inflammation in the synovium and development of arthritis ensue. (5) Antibody-induced cartilage and bone changes, if minor, resolve without any considerable damages. However, if left untreated or in the presence of continuous external stimuli, these changes can give rise to chronic inflammation, joint destruction, and disability [59]. Arthritis is associated with both local as well as systemic pathological manifestations. ACPA: anticitrullinated protein antibody; ADAMTS: a disintegrin and metalloproteinase with thrombospondin motifs; BCR: B cell receptor; CCL: c-c motif chemokine ligand; CXCL: C-X-C motif chemokine ligand; CTLA4: cytotoxic T-lymphocyte-associated protein 4; FcR: Fc receptor; FLS: fibroblast-like synoviocytes; GM-CSF: granulocyte-macrophage colony-stimulating factor; HLA: human leukocyte antigen; IFN- $\gamma$ : interferon gamma; IL: interleukin; IL-1Ra: interleukin-1 receptor antagonist; IL-18BP: interleukin-18-binding protein; LTB ${ }_{4}$ : leukotriene B4; MMP: matrix metalloproteinase; MHC II: major histocompatibility complex class II; NK cell: natural killer cell; PADI: peptidyl arginine deiminase; PDGF: platelet-derived growth factor; PGE $_{2}$ : prostaglandin E2; PTPN22: protein tyrosine phosphatase nonreceptor type 22; RANKL: receptor activator of nuclear factor kappa B ligand; RF: rheumatoid factor; sIL-1RII: soluble interleukin 1 receptor II; sTNFR: soluble tumor necrosis factor receptors; TCR: T cell receptor; TGF $\beta$ : transforming growth factor $\beta$; TNF- $\alpha$ : tumor necrosis factor $\alpha$; TRAF1: TNF receptor-associated factor 1; VEGF: vascular endothelial growth factor. 
of inflammatory mediators. Fibroblasts expressing RANKL together with macrophage colony-stimulating factor (M-CSF) promote differentiation of preosteoclasts into bone-resorbing osteoclasts; this process is initiated at the junction of the cartilage and bone. Targeting $\mathrm{T}$ and $\mathrm{B}$ cells, proinflammatory mediators, signaling molecules, and synovium-specific targets are pursued as new treatment options [5]. In this review, we discuss about disease pathways contributing to cartilage and bone damage to facilitate research on developing targeted drugs.

\section{T Cells}

Increasing evidences demonstrate that RA development results due to an imbalance between $\mathrm{CD} 4^{+} \mathrm{T}$ cell subsets $[6,7]$. Under physiological conditions, $\mathrm{T}$ cells are tolerant toward self-antigens $[8,9]$. The presence of $\mathrm{T}$ cells in the inflamed synovium, association of arthritis with HLA loci, and transfer of disease using $\mathrm{T}$ cells in rodent models suggest the importance of $\mathrm{T}$ cells in arthritis pathogenesis. However, difficulty in identifying consistent $\mathrm{T}$ cell effector mechanisms and not so convincing results from $\mathrm{T}$ celltargeted therapies question this view. Currently, $\mathrm{CD}^{+} \mathrm{T}$ cells reactive with citrulline were identified and the detection rate was high during the first 5 years after RA diagnosis. Although this finding provides a theoretical basis for the correlation between citrulline-specific $\mathrm{CD} 4^{+} \mathrm{T}$ cells and RA, the mechanisms of $\mathrm{T}$ cell activation and its role in promoting joint inflammation need further investigation. Recent studies identified a defective glycolytic process present in T cells from RA patients, which causes glucose to be diverted into the pentose phosphate pathway, driving the accumulation of NADPH and ROS consumption. With an excess of reducing equivalents, $\mathrm{T}$ cells are unable to activate the relevant redox kinase, which enabled bypassing the regulatory checkpoint of the G2/M cell cycle that is conducive for their excessive proliferation [10].

Citrulline-specific $\mathrm{CD}^{+}{ }^{+} \mathrm{T}$ cells of Th1 memory phenotype are higher in RA patients [11]. Upon stimulation with many cytokines, $\mathrm{CD}^{+}{ }^{+} \mathrm{T}$ cells differentiate into Th1, Th2, and Th17 cells secreting different cytokines, while an excessive production of Th17 cells associates with disease severity in many autoimmune diseases. Th17 cells can also mediate osteoclast activation and synovial neovascularization causing bone erosion. Overexpression of Th17-specific transcription factor, retinoic acid-related orphan receptor $(\mathrm{ROR} \gamma \mathrm{t})$, not only induced a high expression of chemokine receptor 6 (CCR6) but also promoted $\mathrm{CD} 4^{+} \mathrm{T}$ cell migration into the affected joints through the CCR6-specific chemokine ligand 20 (CCL20) pathway [7].

On the other hand, Treg cells by secreting IL-10 and TGF- $\beta$ maintain lymphocyte homeostasis and tolerance. When a RA mouse model was administered with sialic acid-binding Ig-like lectin-9, Th17 cell differentiation was reduced and Treg cells proliferated, which attenuated joint inflammation and bone damage. In this context, Foxp3 plays a crucial role by affecting the glycolysis and metabolism of Treg cells through the phosphatidylinositol 3-kinase/protein kinase $\mathrm{B} /$ rapamycin target protein (PI3K/Akt/mTOR) signaling pathway [12].
It has been suggested that PTPN22 encoding a tyrosine phosphatase contributes to the immune tolerance by limiting the signaling events after recognition of autoantigens and weak agonistic antigens by $\mathrm{T}$ cell antigen receptors (TCRs) of naive and effector T cells, while not hindering the response to foreign antigens [13]. This observation suggests that inactivation of the PTPN22 allele can amplify the effector or memory $\mathrm{T}$ cells, which possibly could enhance the development of an autoimmune disease. More importantly, the PTPN22-related alleles have a stronger interaction with arthritis-susceptible HLA-DR alleles. Therefore, an in-depth functional study of PTPN22 gene polymorphisms in arthritis development may further improve our understanding of RA pathogenesis.

\section{Dendritic Cells}

During homeostasis, dendritic cells (DCs) are involved in the maintenance of immune regulation and tolerance. However, in RA by presenting self-peptides, they trigger differentiation and activation of the auto-reactive $\mathrm{T}$ cells as well as innate immune effector functions [14]. In RA patients, increased numbers of DCs are present in the synovial fluid and tissues. Interestingly, tolerogenic DCs (TolDCs) can be generated by genetic and pharmacological modifications or by using cytokines in an antigen-specific manner. Induction of such immune tolerance mechanisms is a promising approach to treat or prevent autoimmune disorders. Many application methods to achieve antigen-specific therapy were reviewed recently [15]. Autologous self-antigen-loaded TolDCs are capable of deleting or reprogramming auto-reactive $\mathrm{T}$ cells and were used for the treatment of experimental arthritis with more promising results [16].

\section{B Cells and Autoantibodies}

Importance of B cells in the pathogenesis of RA has been studied and discussed extensively. Initially, the role of B cells in arthritis was appreciated mainly in terms of autoantibodies because of their importance in clinical diagnosis and prognosis and also as inflammatory mediators. However, B cells can also contribute to disease pathogenesis through antibody-independent mechanisms [17] including antigen presentation, modulation of $\mathrm{T}$ and dendritic cell functions, and production of proinflammatory and regulatory cytokines, facilitating the tertiary lymphoid tissue formation in target organs and possibly tissue repair as well. In recent years, the clinical efficiency of B cell-targeted therapies has revealed the pathogenic properties of B cells clearly in several inflammation-dependent diseases [18]. Recent studies have provided insights into the enrichment of memory B cell subsets distinguished by the expression of Fc-like receptor 4 (FcRL4) in the joints and mucosa-associated lymphoid tissues of RA patients. Interestingly, antibodies produced from FcRL4 ${ }^{+} \mathrm{B}$ cells have high binding capacity to citrullinated autoantigens [19].

Autoantibodies are highly prevalent and detectable in RA patients' sera several years before the clinical symptoms appear [20]. During the time of disease onset, epitope 


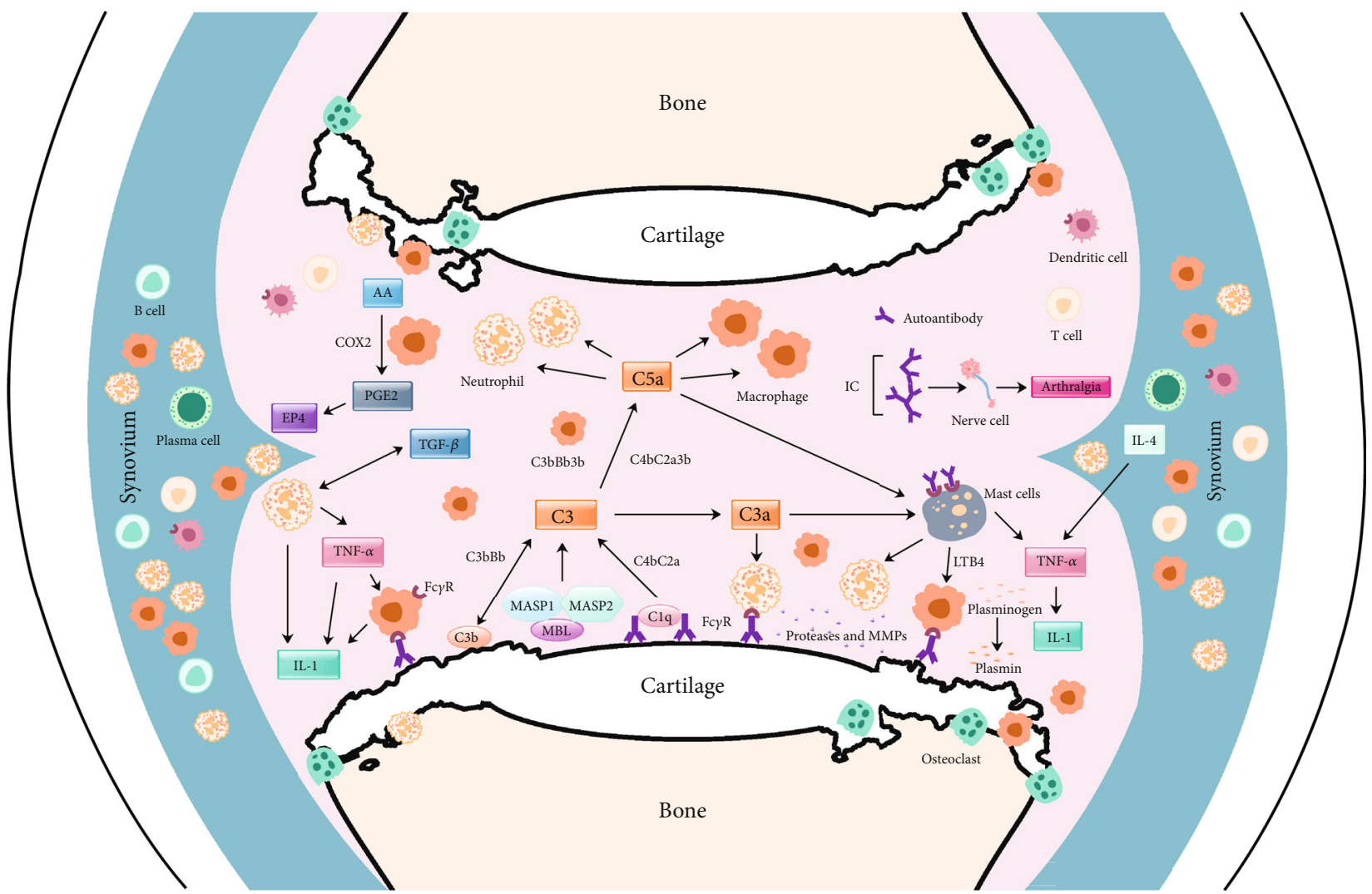

FIGURE 2: Likely interactions of molecules and factors in the antibody mediated joint inflammation. Upon binding to joint antigens or deposited as immune complexes on the cartilage surface, autoantibodies initiate inflammation-dependent and inflammation-independent activities, which culminate in the direct damage to the cartilage and bone. Activation of complement cascades by autoantibodies leads to the release of anaphylatoxins (C3a, C5a), attracting FcR-bearing immune cells to the inflammation foci, which in turn get more activated and secrete cytokines that can further activate resident nonimmune cells in the joint. All these cells in the inflamed joint secrete more inflammatory mediators and extracellular matrix lysing enzymes that could destroy the cartilage and bone. AA: arachidonic acid; C1q, C2a, C3, C3a, C4b and C5a and B (factor B): complement components; CCL3: chemokine (C-C motif) ligand 3; COX2: cyclooxygenase-2; EP4: prostaglandin receptor; MASP: mannose-associated serine protease; MBL: mannose-binding lectin; IC: immune complex; IL: interleukin; LTB4: leukotriene B4; Fc $\gamma$ R: Fc gamma receptors; PGE2: prostaglandin E2; TGF: transforming growth factor; TNF: tumor necrosis factor.

spreading [21], avidity maturation [22] and proinflammatory IgG-Fc glycosylation phenotype [23] of ACPAs were found to occur. Interestingly, the presence of desialylated ACPAs is more during active joint inflammation and transfer of sialic acid-enriched antibodies attenuated experimental arthritis [24]. Alterations in N-glycome induce Fc conformational changes that have direct influence on antibody effector and immunoregulatory functions. Furthermore, germ lineencoded antibodies were identified to be important in experimental arthritis and self-antigen-specific B cells were neither deleted nor anergized [25]. Autoantibodies after binding to their target antigens trigger downstream inflammatory cascades either directly or in the form of immune complexes [26]. At this effector phase of arthritis, activation of different pathways of complement $[27,28]$ and $\mathrm{Fc} \gamma \mathrm{R}$-bearing immune cells contributes to cartilage destruction either directly or by promoting the secretion of inflammatory cytokines and matrix lysing enzymes (Figure 2) [29]. At the same time, antibodies binding to collagen type II (CII) can also induce target damage independent of inflammatory mediators or cells [30]. These antibodies disrupt the integrity of the cartilage matrix by promoting impaired cartilage formation, inhibiting cartilage fibril generation, and disassembling CII fibrils in the ECM. In addition, anti-CII antibodies induced pain prior to and after the appearance of arthritis symptoms and involved in immune complex-mediated activation of neurons [31]. Upon passive transfer, purified antiCII antibodies from RA patients induced arthritis in naive mice [32], which demonstrated their pathogenicity. Unlike anti-CII antibodies, ACPAs might be nonpathogenic [33]. Conversely, ACPAs were shown to mediate osteoclastogenesis [34] and be responsible for bone loss prior to the onset of clinical arthritis [35]; ACPAs induced pain [36] and FLS migration through activation of phosphoinositide 3-kinase [37]. Immune complexes or ACPAs from RA patients induced TNF- $\alpha$ production in peripheral blood mononuclear cells and macrophages [38]. Furthermore, ACPAs were suggested to be agonists for a receptor-mediated response, but this notion is still controversial [39]. Hence, more studies are needed to understand all the possible roles of ACPAs in RA. 


\section{Macrophages}

Macrophages are central in perpetuating arthritis development by stimulating neovascularization, clearing apoptotic immune cells, and promoting the proliferation of fibroblasts and secretion of proteases. Based on the expression of surface molecules, cytokine secretion, and arginine metabolism, macrophages are classified into pro- (M1) and antiinflammatory (M2) phenotypes [40]. The synovial macrophages of RA patients are of M1 phenotype, which highly express proinflammatory proteins PHD3, CCR2, MMP12, and TNF- $\alpha$ with a concomitant low expression M2-type polarization markers [41]. In addition, the level of synuclein A (activin A) encoded by the proinflammatory INHBA gene is significantly elevated in RA patients produced by activated macrophages that can mediate M1-type polarization [42]. Such polarized M1 macrophages secrete a large number of proinflammatory cytokines (IFN- $\gamma$, TNF- $\alpha$, IL- 1 , and IL-6), chemokines (CCL5, CXCL-1, and CXCL-10), and various matrix lysing enzymes, which in turn activate fibroblasts and osteoclasts; aid in the recruitment of neutrophils, monocytes, and lymphocytes; and trigger a series of inflammatory reactions that accelerate inflammation and cause destruction to the articular cartilage. In RA, such a high activation of macrophages increases the expression of toll-like receptors (TLR2, TLR3, TLR4, and TLR7) and promotes the synovial inflammation and cartilage destruction by producing enzymes, cytokines, and other inflammatory factors [43]. In addition, autophagy of macrophages plays an essential role in the pathogenesis of RA [44], which can increase the number of osteoclasts contributing to enhanced bone resorption activity.

\section{Neutrophils}

In RA, neutrophils may alter immune regulation by increasing their cell survival and mobility, having anomalous inflammatory activity, increasing oxidative stress, releasing of neutrophil extracellular traps (NETs), and also by interacting with resident FLS in the synovium to promote inflammatory and antigen-presenting phenotype [45]. High levels of NETs are present in the serum, synovial tissue, rheumatoid nodules, and skin of $\mathrm{ACPA}^{+}$ RA patients. The formation of NETs requires two major biochemical activities. First, the inactivation of the PTPN22 enzyme is considered to be necessary for the production of NETs, which may be involved in the removal of the nuclear envelope and NET components [46]. Second, NETs are composed of DNA and histones, which can be acted upon by peptidyl arginine deiminase type IV (PADI4) causing citrullination. This process prevents histone methylation and transcription leading to chromatin depolymerization, a central event in the NET formation [46]. In addition, NETs contain PAD deposits that promote the formation of citrulline products. Interestingly, the level of NETs in the plasma are highly specific (92\%) and sensitive (91\%) during diagnosis of early RA patients.

\section{Fibroblasts}

Normal FLS in the synovial intimal lining layer has important functions in the maintenance of joint homeostasis by secreting hyaluronan, lubricin, and plasminogen activator; controlling synovial fluid volume and normal inflammatory responses; and regulating leukocyte trafficking and in the maintenance of the joint capsule. In arthritis, FLS are hyperproliferative and an impaired apoptosis could also promote accumulation of FLS in the joints. In RA, FLS produces cytokines and proteases, apart from acquiring an aggressive, tumor-like phenotype because of transcriptional mechanisms of imprinting and epigenetic changes, which could mediate cartilage destruction and drive joint inflammation [47]. IL-17 is one of the crucial factors in transforming FLS into an invasive RA-FLS type and may directly assist in FLS-mediated progression of RA by significantly increasing its activation, migration, and invasive potential. RA-FLS also secretes many proangiogenic factors like fibroblast growth factor, vascular endothelial growth factor (VEGF), hypoxiainducible factors (HIFs), and IL-18, which promote new blood vessel formation, pannus growth, and inflammation.

\section{Chondrocytes}

Chondrocytes are unique to the articular cartilage, which maintain an equilibrium between synthesis and breakdown of extracellular matrix under physiological conditions. A chondrocyte secretome contains extracellular matrix proteins, cytokines, growth factors, enzymes, and their inhibitors as well as many other protein components having different target specificities [48]. Cytokines trigger chondrocytes to release more cytokines and matrix metalloproteinases (MMPs) that can degrade the cartilage and also inhibit generation of tissue inhibitors of metalloproteinases (TIMPs). IL- $1 \beta$ released during inflammation can increase the catabolic activities of chondrocytes by inhibiting the spontaneous calcium signaling as well as in altering signaling in the cell cycle and Rho GTPases present within the chondrocytes. Dependent on the release of proinflammatory cytokines from the synovium, chondrocytes are activated to participate in cartilage damage. At the same time, they could also act as the source of proinflammatory cytokines, which in turn increases catabolic events in the cartilage while suppressing anabolic tissue repair and remodeling processes.

\section{Role of Cytokines}

Cytokines are involved in many inflammatory events related with the regulation of inflammation, autoimmune responses, synovitis, and articular joint destruction. Many crucial cytokines (IL-1, IL-6, IL-10, IL-12, IL-15, IL-17, IL-18, TNF- $\alpha$, TGF $\beta$, IL-23, etc.) [49] (Table 1) and all the four family of chemokines (CXC, CC, C, and $\mathrm{CX}_{3} \mathrm{C}$ ) [50] (Table 2) are contributing to the joint inflammation. Successful amelioration of signs and symptoms of arthritis in patients with TNF- $\alpha$ neutralizing agents has transformed RA treatment strategies quite significantly, which facilitated further research to target other inflammatory cytokines like IL-1, IL-6, and IL-17. 


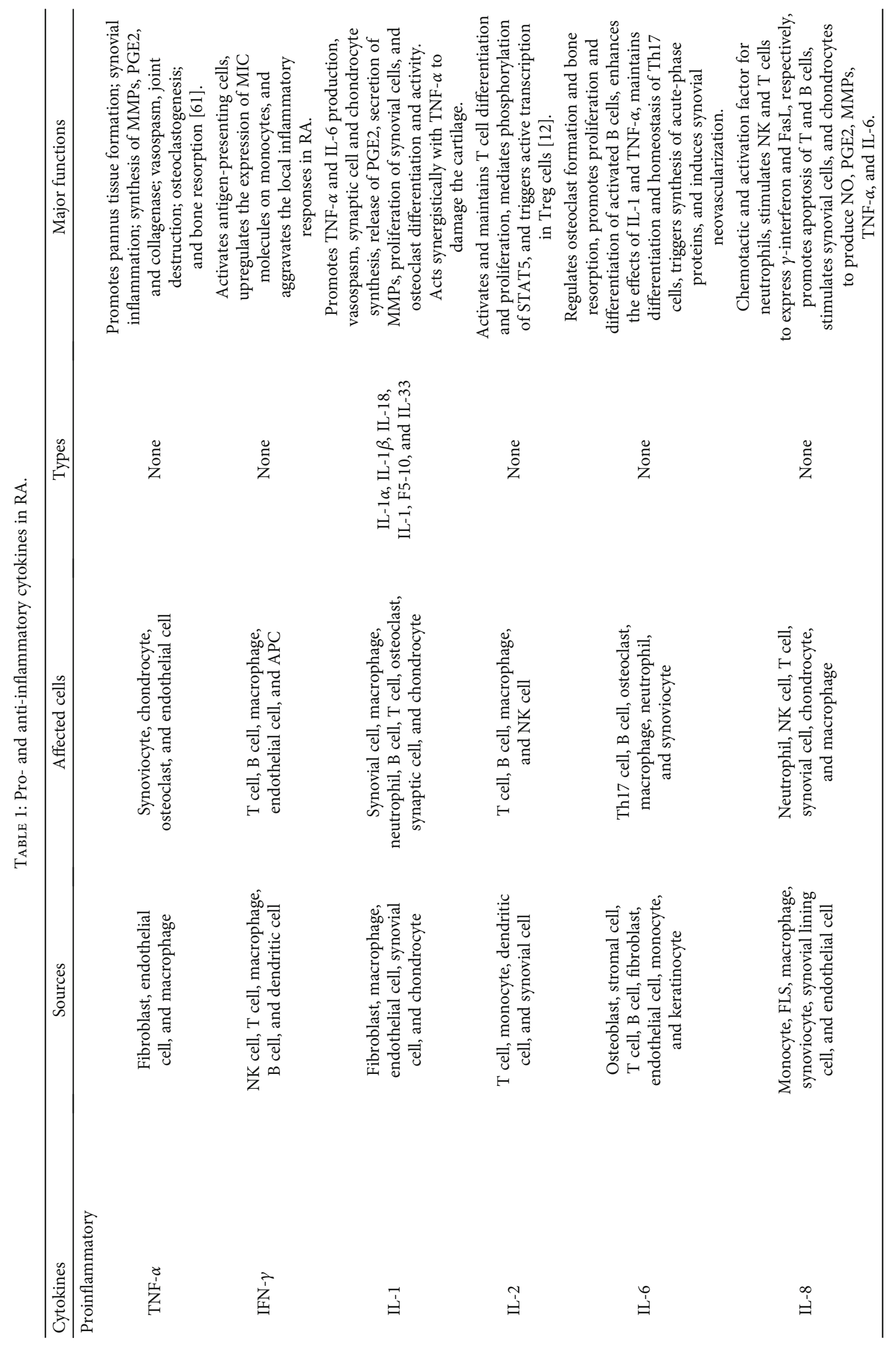









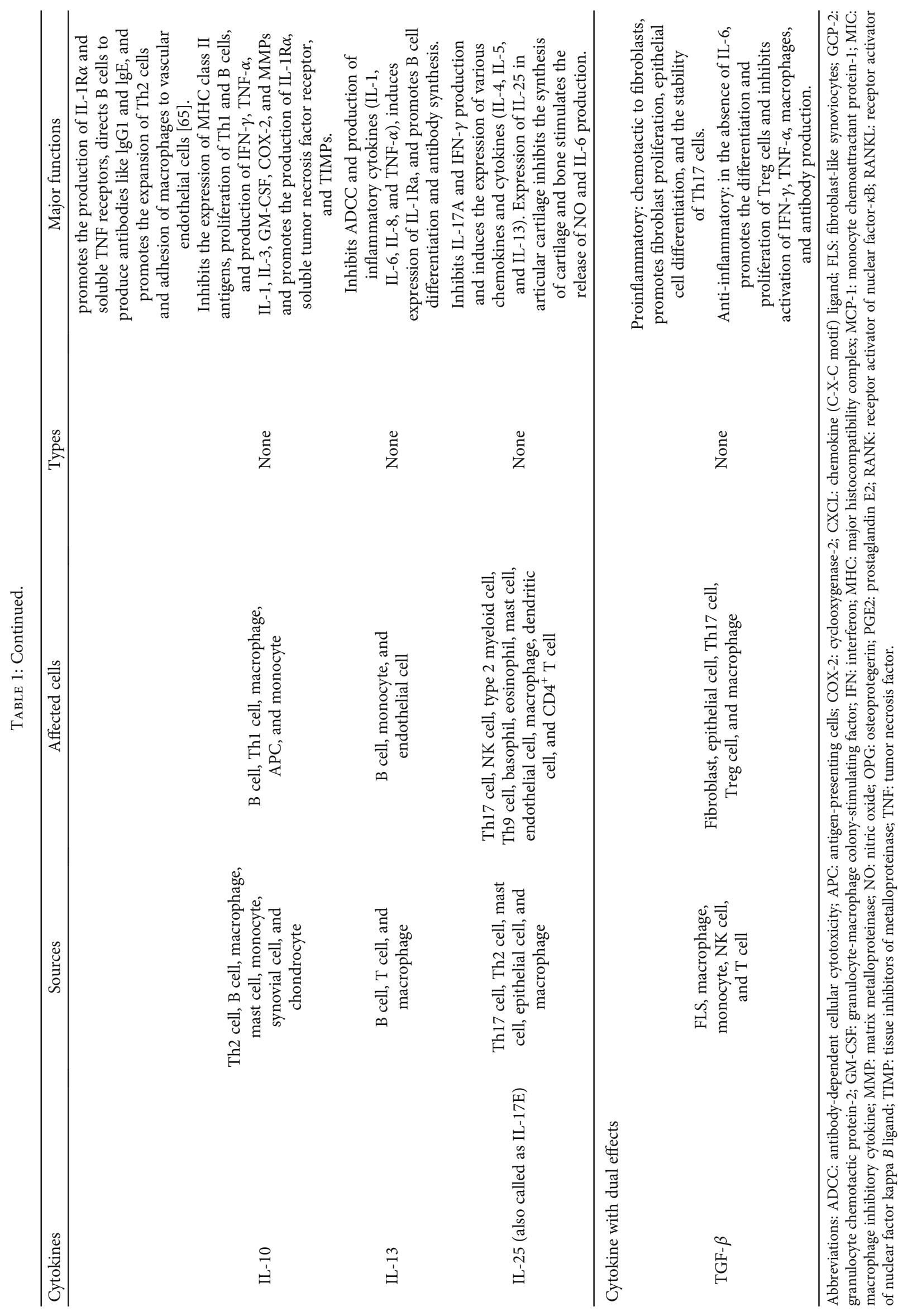









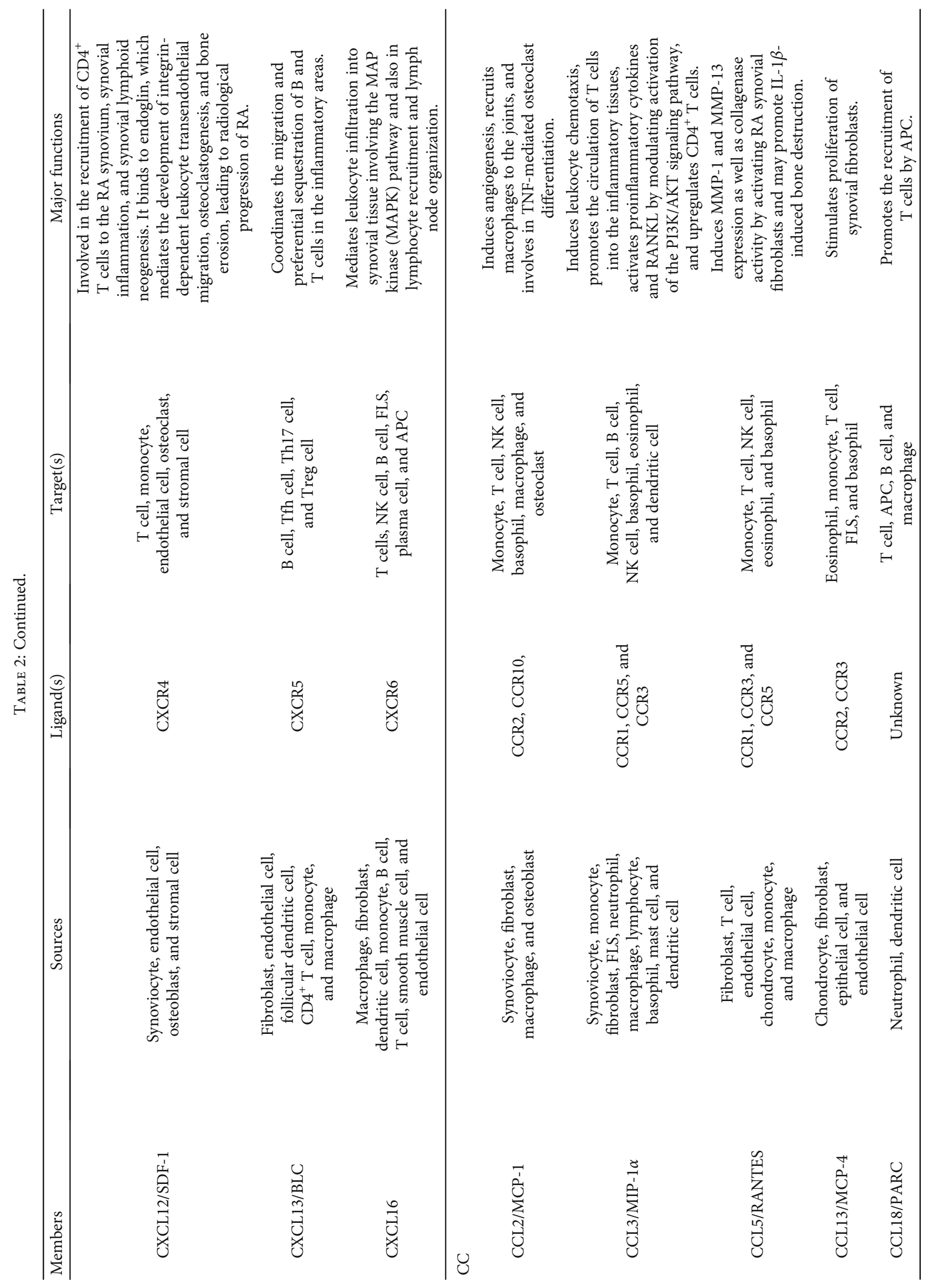




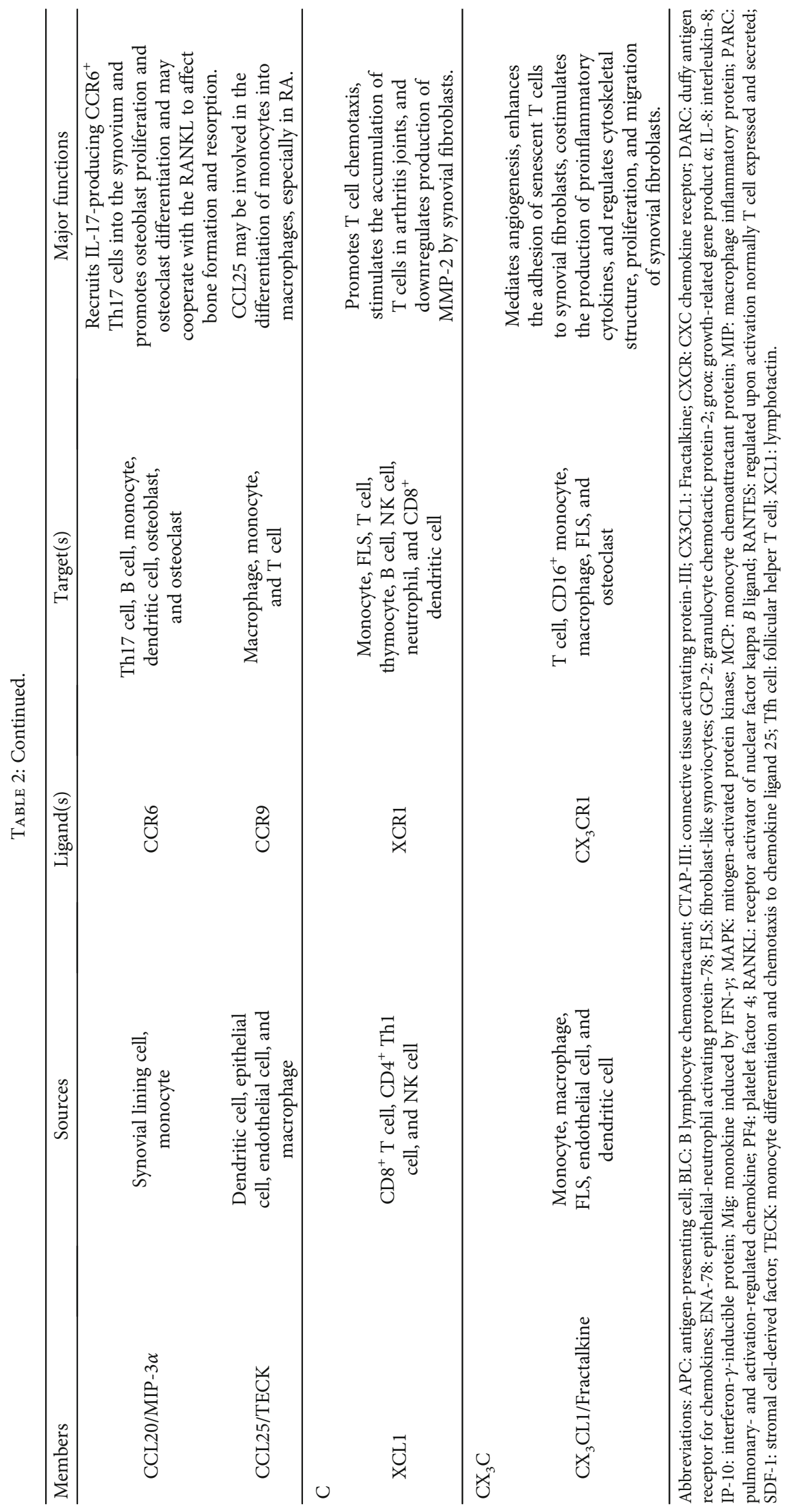


However, targeting cytokines should be done with caution because they are pleiotropic, redundant, and multifunctional apart from having antagonistic and synergistic functions between them. Many RA patients are still reported to be refractory to anti-TNF- $\alpha$ therapy and TNF inhibitors were found to be more effective in clinical trials than in daily clinical practice. Moreover, anti-inflammatory cytokines also can promote joint inflammation [51]. Pro- and anti-inflammatory cytokines and chemokines involved in joint inflammation/resolution, their cellular sources, target cells, and major functions are summarized in Tables 1 and 2 , respectively.

\section{MMPs, ADAMTS, and TIMPs}

Several proteinases like MMPs, ADAMTS (a disintegrin and metalloproteinase with thrombospondin motifs), neutrophil elastase, and cathepsins ( $\mathrm{G}$ and $\mathrm{B}$ ) can damage the cartilage directly. Depletion of proteoglycans from articular cartilage is an initial event in RA development leading to the degradation of the collagen fibrils. It is of interest to note that CIIreactive monoclonal antibodies upon passive transfer induced a significant amount of proteoglycan depletion within 72 hours [30]. Many MMPs (MMP 1-3, MMP 7-9, MMP-13, and MT1-MMP) preferentially split the bond between $\mathrm{Asn}^{341}$-Phe ${ }^{342}$ of aggrecan. Conversely, ADAMTS1, ADAMTS4, and ADAMTS5 cleave the $\mathrm{Glu}^{373}$ - $\mathrm{Ala}^{374}$ bond in addition to other sites in the G2-G3 domains of proteoglycans. Thus, both the MMP and ADAMTS enzymes contribute to aggrecan degradation during arthritis development. TIMPs are endogenous blockers of MMPs and regulators of matrix turnover, tissue reorganization, and cellular activity. Sources, targets, and receptors/ligands and major functions of MMPs, ADAMTS, and TIMPs involved in arthritis pathogenesis are summarized in Table 3.

\section{Signaling Pathways Affecting Bone Destruction}

Bone erosion starts during the early phase of arthritis development causing deformity of the articular joints, which affects quality of patients' life. Molecular mechanisms underlying differentiation and activation of bone-eroding cells, osteoclasts, are well documented, and several signaling pathways are contributing to osteoclast maturation and activation causing joint destruction (Figure 3).

\section{RANKL/RANK Pathway}

RANKL (also called TNFSF11, OPGL, TRANCE, and ODF) and its receptor RANK are indispensable regulators of bone repair and remodeling processes. Several hormones and cytokines induce RANKL production in osteoblasts and synovial fibroblasts. After binding with RANK, RANKL triggers the recruitment of an adaptor molecule TRAF-6 resulting in the activation of signaling molecules like NF$\kappa \mathrm{B}$, c-Jun N-terminal kinase (JNK), AKT/PKB, ERK, Src, and p38 mitogen-activated protein (MAP) kinases and the transcription factor, and nuclear factor of activated $\mathrm{T}$ cells, calcineurin-dependent 1 (NFATc1) [52]. Hence, the RANKL/RANK signaling pathway is a potential therapeutic target in osteolytic diseases. Denosumab (RANKL-specific human monoclonal antibody) is currently used for treating osteoporosis, osteosarcoma, multiple myeloma, and bone metastasis [53]. Although denosumab is highly specific to RANKL and has a good effect on bones, safety concerns still exist. On the other hand, the RANKL/RANK pathway is having important functions in osteoblasts as well. Vesicular RANK, secretion product of matured osteoclasts, by binding to osteoblast-derived RANKL facilitates bone formation by initiating RANKL reverse signaling leading to the activation of Runt-related transcription factor 2 (Runx2) [54].

\section{NF- $\kappa$ B Signaling Pathway}

Initiation of the RANKL/RANK pathway causes NF- $\kappa \mathrm{B}$ activation, which contributes to osteoclast differentiation. After NF- $\kappa$ B stimulation, several TNF-receptor- (TNFR-) related factors associate with the cytoplasmic domain of RANK. Among them, TRAF-6 is indispensable for osteoclast formation and activation [55], while NF- $\kappa \mathrm{B}$ p50 and p52 subunits modulate RANKL and TNF- $\alpha$-induced differentiation of osteoclast precursors. Mice deficient in p50 and p52 proteins are osteopetrotic. NF- $\kappa \mathrm{B}$-activating upstream catalytic (IKK- $\alpha$ and IKK- $\beta$ ) and noncatalytic (IKK- $\gamma$ also known as NEMO) subunits of I $\kappa \mathrm{B}$ kinase are also crucial in the generation of osteoclasts. RelB is the NF- $\kappa \mathrm{B}$-inducing kinase (NIK) downstream subunit, which is also responsible for osteoclast differentiation.

\section{MAPKs}

Mitogen-activated protein kinase (MAPK) lineage consists of p38-MAP kinases (p38-MAPK $\alpha, \beta, \gamma$, and $\delta$ isoforms), c-Jun $\mathrm{N}$-terminal kinases (JNK1-3), and extracellular signalregulated kinases (ERK1-2). RANKL stimulation activates many of these kinases, which regulate different cellular responses. When a specific inhibitor (SB203580) or a natural product from teasel for $\mathrm{p} 38$-MAPK $\alpha$ and $\beta$ was used, significant inhibition of osteoclast formation but not its functions was observed. RANKL-activated p38-MAPKs can directly phosphorylate STAT1 and regulate the expression of target genes. In addition, JNKs and their upstream kinase MKK7 are also involved in osteoclastogenesis. However, neither JNK1 nor JNK2 deficiency led to significant bone defects. Mice deficient in both JNK1 and JNK2 have embryonic lethality during midgestation [56]; hence, a conditional knock-out in the bone marrow might address the importance of JNKs in osteoclastogenesis. AP-1 and related genes (c-Jun, JunB, c-Fos, and Fra but not JunD), controlled by JNKs, are also crucial for osteoclast differentiation and maturation. ERK is another MAPK subunit getting activated upon RANKL stimulation, which regulates the survival and differentiation of both osteoclasts and osteoblasts. However, several receptor systems in many organs operate via NF- $\kappa \mathrm{B}$ and MAPK pathways; hence, targeting these pathways might not be optimal for treating bone damage. 


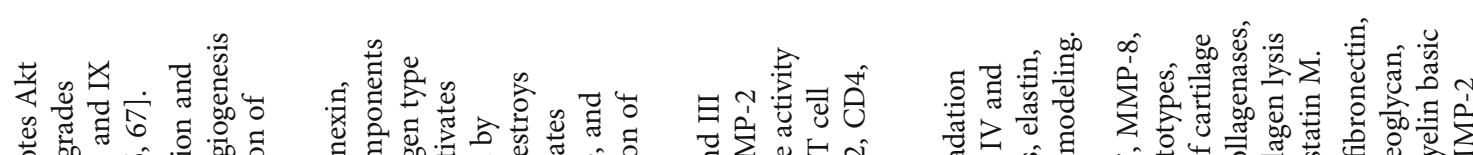

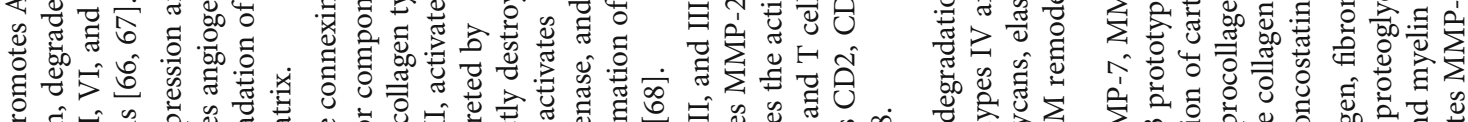







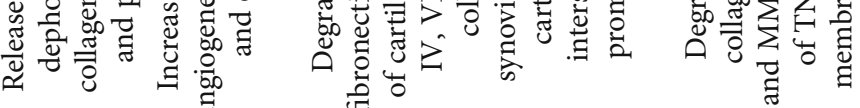
:



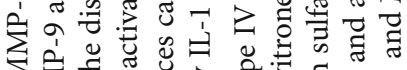

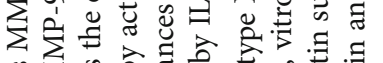

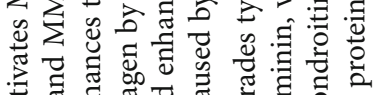

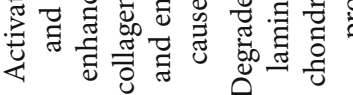

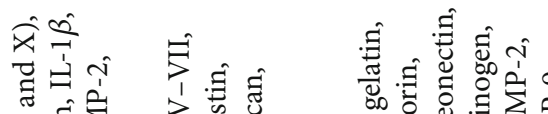



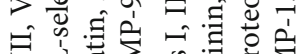

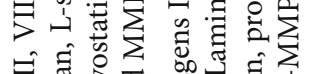

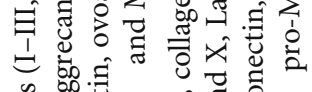

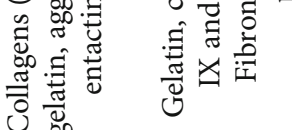

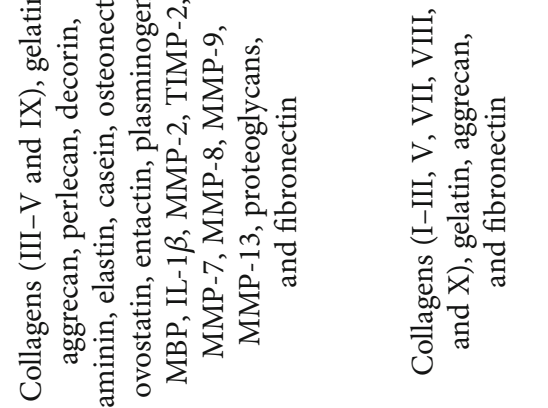



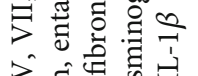



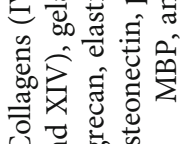

ठ용

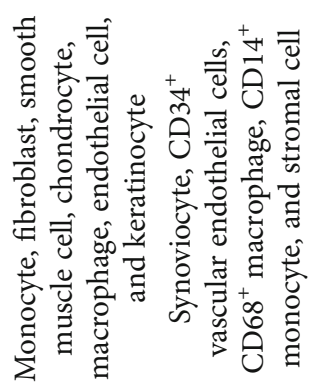





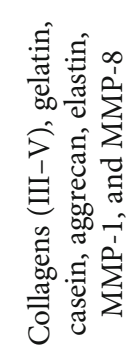
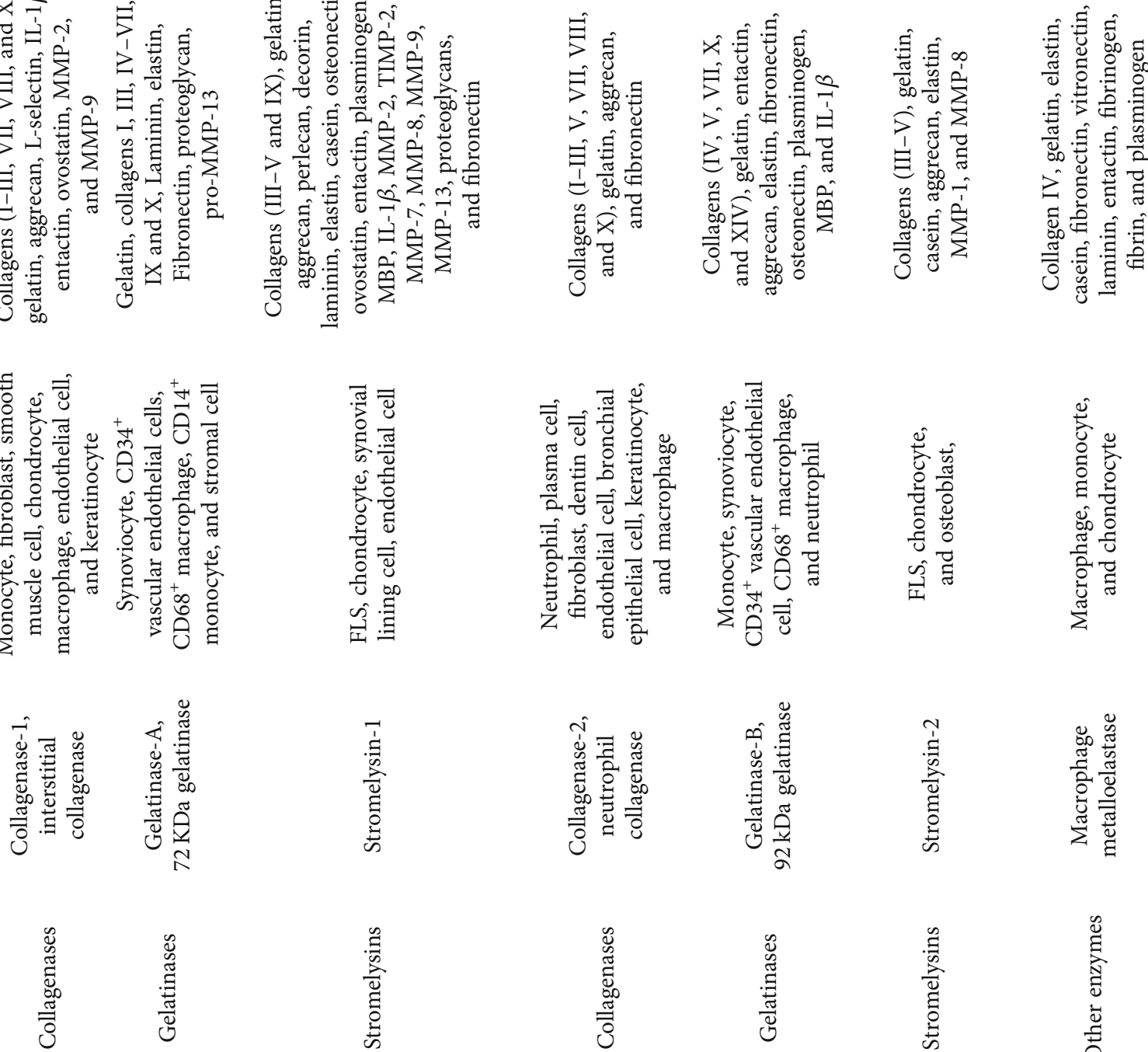

:
:
:
离
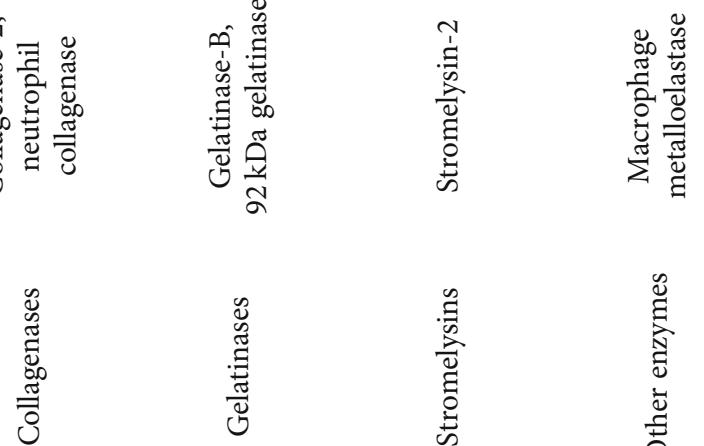

$\sum_{i}^{1} \quad \sum_{i}^{1}$

$\sum_{i}^{m}$

$\sum_{\Sigma}^{\infty} \quad \sum$

$\sum_{i}^{i} \quad \stackrel{0}{\dot{1}}$

$\sum_{i}^{71}$ 


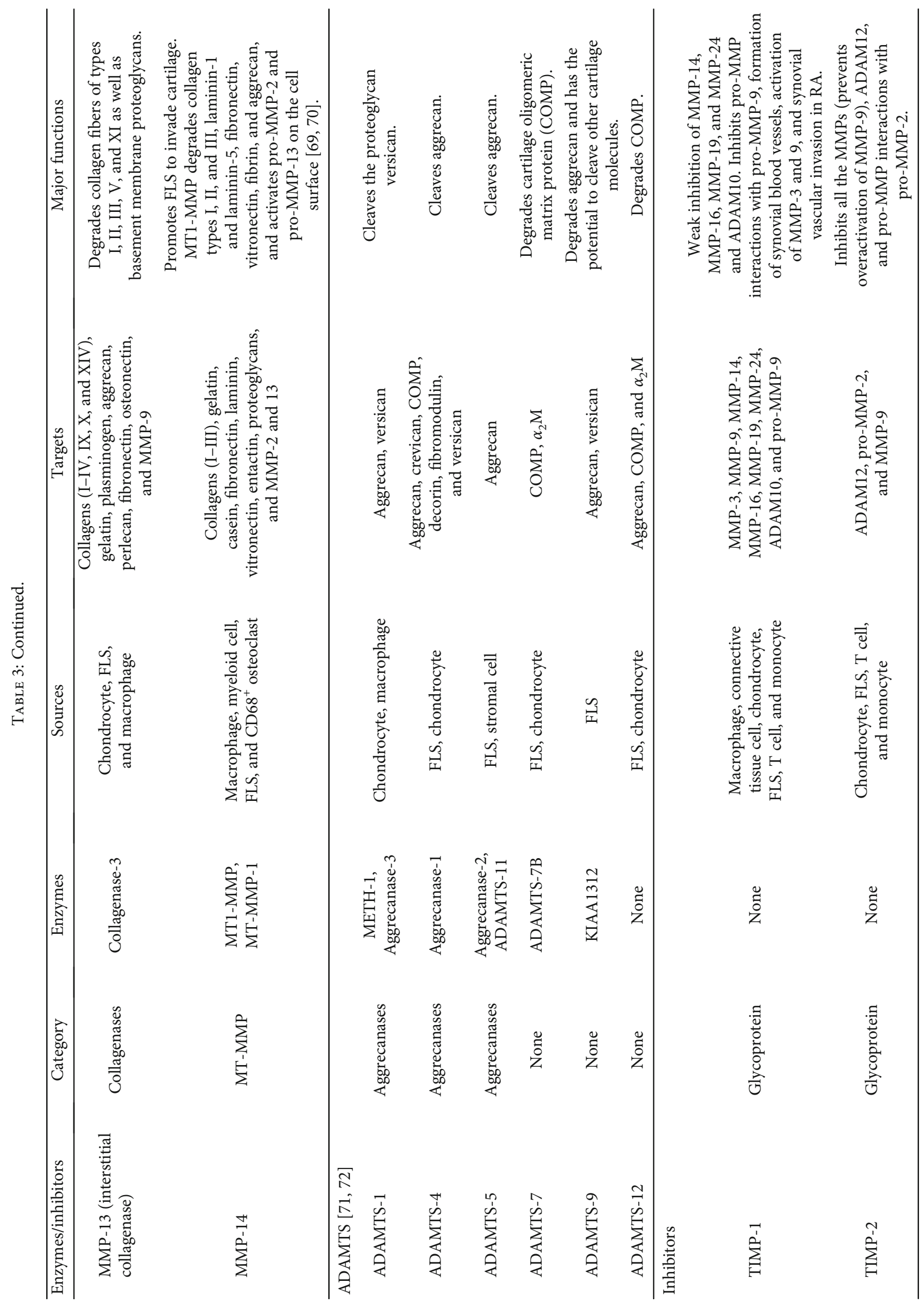




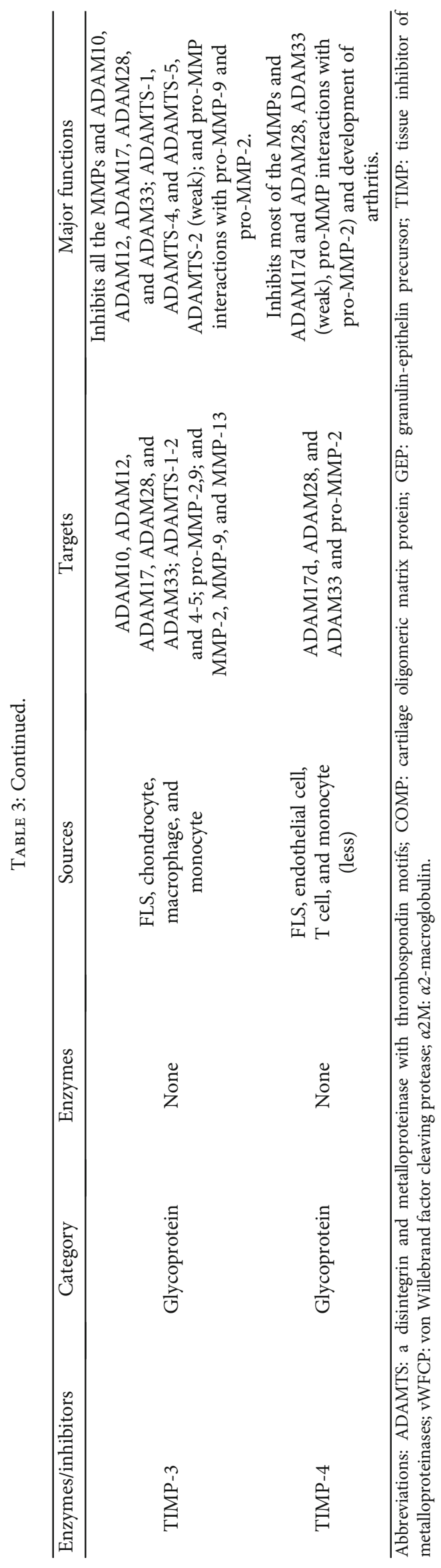



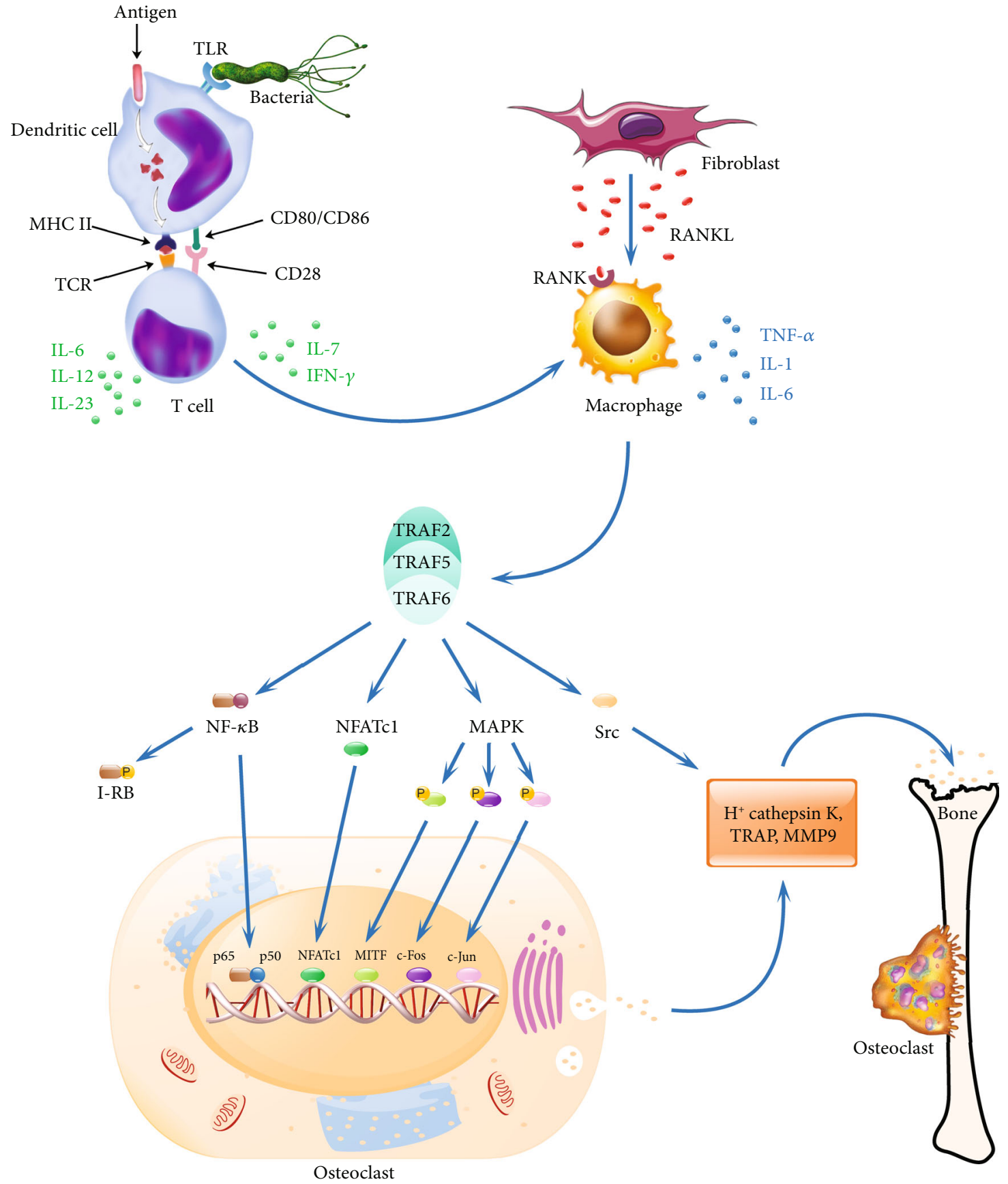

FIgURE 3: Signaling pathways in osteoclast activation. During RA pathogenesis, antigen-presenting cells after uptake of an autoantigen or pathogenic molecules process and present antigenic determinants on their cell surface in conjunction with arthritis-permissible HLA/MHC class II molecules, which activate differentiation of T cells into different subphenotypes. The activated T cells secrete various cytokines like IL-6, IL-7, IL-10, IL-12, IL-17, IL-23, and IFN- $\gamma$. These cytokines modulate macrophages to secrete various pro- and/or anti-inflammatory cytokines and other inflammatory mediators. Upon exposure to the inflammatory cytokines, fibroblast-like synoviocytes express RANKL, which binds with its receptor (RANK) present on the cell surface of activated macrophages initiating the RANK/RANKL pathway through TRAF 2, 5, and 6 proteins, which leads to the activation of downstream NF- $\kappa$ B, MAPK, NFATc1, and Src signaling cascades. These factors after translocation initiate the expression of genes like TRAP, CtsK, and MMP-9 in the nucleus, which promote osteoclastogenesis and bone resorption. TLR: toll-like receptor; TCR: T cell receptor; CtsK: cathepsin K; I- $\kappa \mathrm{B}$ : inhibitor of the NF- $\kappa$ B transcription factor; IL: interleukin; IFN- $\gamma$ : interferon gamma; MAPK: mitogen-activated protein kinase; MHC II: major histocompatibility complex II; MITF: microphthalmia-associated transcription factor; MMP 9: matrix metalloproteinase 9; NFATc1: nuclear factor of activated T cells, calcineurin-dependent 1 ; NF- $\kappa$ B: nuclear factor kappa B; p50 and p65: REL-associated proteins (also called NF- $\kappa$ B1 and RelA) involved in NF- $\kappa$ B heterodimer formation and nuclear translocation and activation; RANK: receptor activator of nuclear factor $-\kappa \mathrm{B}$; RANKL: receptor activator of nuclear factor $\kappa \mathrm{B}$ ligand; Src: intracellular non-receptor tyrosine kinase; TRAF: TNF receptor-associated factor; TRAP: tartrate-resistant acid phosphatase. c-Jun and c-Fos form the early response transcription factor, AP-1. 


\section{NFATc1}

Stimulating with RANKL leads to the induction of several genes mediating osteoclast differentiation and function, including cellular fusion, polarization, and secretion of acid hydrolases. Some of these genes are transcribed by another key factor, NFATc1 [57], which is a downstream target of RANKL. NFATc1 expression causes osteoclast differentiation and induction of osteoclast-related genes including TRAP, cathepsin $\mathrm{K}$, and calcitonin receptors in association with c-Fos. NFATc1-deficient cells are defective in osteoclastogenesis. In the absence of RANKL, overexpression of NFATc1 induced osteoclast precursor cell differentiation into $\mathrm{TRAP}^{+}$osteoclast-like cells. Moreover, c-Jun signaling is also critical in the regulation of NFATc1 activation. Transgenic mice specifically expressing dominant-negative form of c-Jun in the osteoclasts exhibit severe osteopetrosis. Thus, NFATc1 acts as an important mediator in coupling RANK signaling events to osteoclast differentiation.

\section{Src Kinase}

Src is a protein-tyrosine kinase involved in the cell development, division, relocation, and survival. The resorption process of osteoclasts depends on their attachment and movement on the surface of the bones to form a sealing zone [58]. Targeted interference in c-Src gene expression led to the development of osteopetrosis, and it is a critical factor in RANKL-induced activation of protein tyrosine kinase 2 (Pyk2) and $\alpha \mathrm{v} \beta 3$ integrin assembly, which is essential for the adhesion and skeleton organization. Binding of RANK and RANKL meditates the recruitment of TRAF- 6 and cSrc. Subsequently, TRAF- 6 enhances c-Src activation causing phosphorylation of signaling molecules like E3 ubiquitinprotein ligase, and Cbl. Src complexes with Pyk2, Cbl, and ADAP (adhesion and degranulation promoting adaptor protein, also called SLAP-130 or Fyb). Phosphorylation of these signaling molecules is a prerequisite for integrin-mediated osteoclast functions. Hence, targeting $\mathrm{c}$-Src might be a viable future treatment strategy for osteoporosis and higher bone resorption observed in RA patients.

\section{Conclusions}

Interplay between multiple factors engender aberrations in immune recognition and activation causing initiation of molecular pathways targeting cartilage and bone. Various immune and nonimmune cells are crucial during this process. Resident and infiltrating cells in the joints proliferate and secrete proinflammatory cytokines, chemokines, and matrix lysing enzymes that could destroy the joints leading to functional loss. Moreover, different signaling cascades are activated during osteoclast activation and differentiation that are involved in the bone resorption activity. Hence, targeting a single effector molecule is insufficient to block cartilage and bone damage in arthritis. Since RA is an immune-mediated disorder, therapeutics restoring immune balance certainly can improve clinical therapy.

\section{Additional Points}

Key Messages. Rheumatoid arthritis is a multi-factorial syndrome involving interactions between genetic, epigenetic and environmental factors. Initiation events damaging joints start very early during the autoimmune phase of arthritis development. Comprehensive knowledge on pathogenesis is a prerequisite for developing optimal treatment and potential drugs. Targeting a single effector molecule is insufficient to block cartilage and bone damage in arthritis. Since rheumatoid arthritis is an immune-mediated disorder, therapeutics restoring immune balance can improve clinical therapy.

\section{Conflicts of Interest}

The authors declare no conflict of interest.

\section{Authors' Contributions}

All the three authors contributed in preparing this manuscript.

\section{Acknowledgments}

KSN would like to thank the Southern Medical University, Guangzhou, China, for the start-up grants (C1034211, C1051004) and international exploration grant (C1051427).

\section{References}

[1] I. B. McInnes and G. Schett, "Pathogenetic insights from the treatment of rheumatoid arthritis," Lancet, vol. 389, no. 10086, pp. 2328-2337, 2017.

[2] C. Orr, E. Vieira-Sousa, D. L. Boyle et al., "Erratum: Synovial tissue research: a state-of-the-art review," Nature Reviews Rheumatology, vol. 13, no. 10, p. 630, 2017.

[3] Q. Fang, J. Ou, and K. S. Nandakumar, "Autoantibodies as diagnostic markers and mediator of joint inflammation in arthritis," Mediators of Inflammation, vol. 2019, Article ID 6363086, 22 pages, 2019.

[4] U. Harre, S. C. Lang, R. Pfeifle et al., "Glycosylation of immunoglobulin $\mathrm{G}$ determines osteoclast differentiation and bone loss," Nature Communications, vol. 6, no. 1, p. 6651, 2015.

[5] G. R. Burmester, E. Feist, and T. Dorner, "Emerging cell and cytokine targets in rheumatoid arthritis," Nature Reviews Rheumatology, vol. 10, no. 2, pp. 77-88, 2014.

[6] K. Yudoh, H. Matsuno, F. Nakazawa, T. Yonezawa, and T. Kimura, "Reduced expression of the regulatory CD4+ T cell subset is related to Th1/Th2 balance and disease severity in rheumatoid arthritis," Arthritis and Rheumatism, vol. 43, no. 3, pp. 617-627, 2000.

[7] N. Komatsu, K. Okamoto, S. Sawa et al., "Pathogenic conversion of Foxp 3+ T cells into TH17 cells in autoimmune arthritis," Nature Medicine, vol. 20, no. 1, pp. 62-68, 2014.

[8] V. Malmstrom, J. Backlund, L. Jansson, J. Kihlberg, and R. Holmdahl, "T cells that are naturally tolerant to cartilagederived type II collagen are involved in the development of collagen-induced arthritis," Arthritis Research, vol. 2, no. 4, pp. 315-326, 2000.

[9] M. J. van Herwijnen, L. Wieten, R. van der Zee et al., "Regulatory $\mathrm{T}$ cells that recognize a ubiquitous stress-inducible self- 
antigen are long-lived suppressors of autoimmune arthritis," Proceedings of the National Academy of Sciences, vol. 109, no. 35, pp. 14134-14139, 2012.

[10] Z. Yang, Y. Shen, H. Oishi et al., "Restoring oxidant signaling suppresses proarthritogenic $\mathrm{T}$ cell effector functions in rheumatoid arthritis," Science Translational Medicine, vol. 8, no. 331, pp. 331ra38-331ra38, 2016.

[11] E. A. James, M. Rieck, J. Pieper et al., "Citrulline-specific Th1 cells are increased in rheumatoid arthritis and their frequency is influenced by disease duration and therapy," Arthritis \& Rhematology, vol. 66, no. 7, pp. 1712-1722, 2014.

[12] L. Lu, J. Barbi, and F. Pan, "The regulation of immune tolerance by FOXP3," Nature Reviews Immunology, vol. 17, no. 11, pp. 703-717, 2017.

[13] R. J. Salmond, R. J. Brownlie, V. L. Morrison, and R. Zamoyska, "The tyrosine phosphatase PTPN22 discriminates weak self peptides from strong agonist TCR signals," Nature Immunology, vol. 15, no. 9, pp. 875-883, 2014.

[14] P. Wehr, H. Purvis, S. C. Law, and R. Thomas, "Dendritic cells, T cells and their interaction in rheumatoid arthritis," Clinical and Experimental Immunology, vol. 196, no. 1, pp. 12-27, 2019.

[15] A. K. Shakya and K. S. Nandakumar, "Antigen-specific tolerization and targeted delivery as therapeutic strategies for autoimmune diseases," Trends in Biotechnology, vol. 36, no. 7, pp. 686-699, 2018.

[16] K. Schinnerling, L. Soto, P. Garcia-Gonzalez, D. Catalan, and J. C. Aguillon, "Skewing dendritic cell differentiation towards a tolerogenic state for recovery of tolerance in rheumatoid arthritis," Autoimmunity Reviews, vol. 14, no. 6, pp. 517-527, 2015.

[17] P. Shen and S. Fillatreau, "Antibody-independent functions of B cells: a focus on cytokines," Nature Reviews Immunology, vol. 15, no. 7, pp. 441-451, 2015.

[18] K. Hofmann, A. K. Clauder, and R. A. Manz, "Targeting B cells and plasma cells in autoimmune diseases," Frontiers in Immunology, vol. 9, p. 835, 2018.

[19] Y. Tanaka and T. Ohira, "Mechanisms and therapeutic targets for bone damage in rheumatoid arthritis, in particular the RANK-RANKL system," Current Opinion in Pharmacology, vol. 40, pp. 110-119, 2018.

[20] S. Rantapää-Dahlqvist, B. A. W. de Jong, E. Berglin et al., "Antibodies against cyclic citrullinated peptide and IgA rheumatoid factor predict the development of rheumatoid arthritis," Arthritis \& Rheumatism, vol. 48, no. 10, pp. 2741-2749, 2003.

[21] D. van der Woude, S. Rantapaa-Dahlqvist, A. Ioan-Facsinay et al., "Epitope spreading of the anti-citrullinated protein antibody response occurs before disease onset and is associated with the disease course of early arthritis," Annals of the Rheumatic Diseases, vol. 69, no. 8, pp. 1554-1561, 2010.

[22] P. Suwannalai, L. A. van de Stadt, H. Radner et al., "Avidity maturation of anti-citrullinated protein antibodies in rheumatoid arthritis," Arthritis \& Rheumatism, vol. 64, no. 5, pp. 1323-1328, 2012.

[23] Y. Rombouts, E. Ewing, L. A. van de Stadt et al., "Anti-citrullinated protein antibodies acquire a pro-inflammatory Fc glycosylation phenotype prior to the onset of rheumatoid arthritis," Annals of the Rheumatic Diseases, vol. 74, no. 1, pp. 234-241, 2015.
[24] Y. Ohmi, W. Ise, A. Harazono et al., "Sialylation converts arthritogenic IgG into inhibitors of collagen-induced arthritis," Nature Communications, vol. 7, no. 1, article 11205, 2016.

[25] D. Cao, I. Khmaladze, H. Jia et al., "Pathogenic autoreactive B cells are not negatively selected toward matrix protein collagen II," Journal of Immunology, vol. 187, no. 9, pp. 4451-4458, 2012.

[26] R. J. Ludwig, K. Vanhoorelbeke, F. Leypoldt et al., "Mechanisms of autoantibody-induced pathology," Frontiers in Immunology, vol. 8, p. 603, 2017.

[27] E. A. Bemis, J. M. Norris, J. Seifert et al., "Complement and its environmental determinants in the progression of human rheumatoid arthritis," Molecular Immunology, vol. 112, pp. 256-265, 2019.

[28] V. M. Holers and N. K. Banda, "Complement in the initiation and evolution of rheumatoid arthritis," Frontiers in Immunology, vol. 9, p. 1057, 2018.

[29] K. S. Nandakumar, "Targeting IgG in arthritis: disease pathways and therapeutic avenues," International Journal of Molecular Sciences, vol. 19, no. 3, p. 677, 2018.

[30] K. S. Nandakumar, E. Bajtner, L. Hill et al., "Arthritogenic antibodies specific for a major type II collagen triple-helical epitope bind and destabilize cartilage independent of inflammation," Arthritis and Rheumatism, vol. 58, no. 1, pp. 184196, 2008.

[31] A. B. Farinotti, G. Wigerblad, D. Nascimento et al., "Cartilagebinding antibodies induce pain through immune complexmediated activation of neurons," The Journal of Experimental Medicine, vol. 216, no. 8, pp. 1904-1924, 2019.

[32] S. B. Petkova, K. N. Konstantinov, T. J. Sproule, B. L. Lyons, M. A. Awwami, and D. C. Roopenian, "Human antibodies induce arthritis in mice deficient in the low-affinity inhibitory IgG receptor Fc gamma RIIB," The Journal of Experimental Medicine, vol. 203, no. 2, pp. 275-280, 2006.

[33] H. Yamada, T. Ozawa, H. Kishi et al., "Cutting edge: B cells expressing cyclic citrullinated peptide-specific antigen receptor are tolerized in normal conditions," Journal of Immunology, vol. 201, no. 12, pp. 3492-3496, 2018.

[34] U. Harre, D. Georgess, H. Bang et al., "Induction of osteoclastogenesis and bone loss by human autoantibodies against citrullinated vimentin," The Journal of Clinical Investigation, vol. 122, no. 5, pp. 1791-1802, 2012.

[35] A. Kleyer, S. Finzel, J. Rech et al., "Bone loss before the clinical onset of rheumatoid arthritis in subjects with anticitrullinated protein antibodies," Annals of the Rheumatic Diseases, vol. 73, no. 5, pp. 854-860, 2014.

[36] G. Wigerblad, D. B. Bas, C. Fernades-Cerqueira et al., "Autoantibodies to citrullinated proteins induce joint pain independent of inflammation via a chemokine-dependent mechanism," Annals of the Rheumatic Diseases, vol. 75, no. 4, pp. 730-738, 2016.

[37] M. Sun, B. Rethi, A. Krishnamurthy et al., "Anticitrullinated protein antibodies facilitate migration of synovial tissuederived fibroblasts," Annals of the Rheumatic Diseases, vol. 78, no. 12, pp. 1621-1631, 2019.

[38] L. Laurent, C. Clavel, O. Lemaire et al., "Fc $\gamma$ receptor profile of monocytes and macrophages from rheumatoid arthritis patients and their response to immune complexes formed with autoantibodies to citrullinated proteins," Annals of the Rheumatic Diseases, vol. 70, no. 6, pp. 1052-1059, 2011. 
[39] R. Toes and D. S. Pisetsky, "Pathogenic effector functions of ACPA: where do we stand?," Annals of the Rheumatic Diseases, vol. 78, no. 6, pp. 716-721, 2019.

[40] J. B. Self-Fordham, A. R. Naqvi, J. R. Uttamani, V. Kulkarni, and S. Nares, "MicroRNA: Dynamic regulators of macrophage polarization and plasticity," Frontiers in Immunology, vol. 8, p. 1062, 2017.

[41] B. S. Palacios, L. Estrada-Capetillo, E. Izquierdo et al., "Macrophages from the synovium of active rheumatoid arthritis exhibit an activin A-dependent pro-inflammatory profile," The Journal of Pathology, vol. 235, no. 3, pp. 515-526, 2015.

[42] J. Massague, "TGF $\beta$ signalling in context," Nature Reviews Molecular Cell Biology, vol. 13, no. 10, pp. 616-630, 2012.

[43] C. M. Cuda, R. M. Pope, and H. Perlman, "The inflammatory role of phagocyte apoptotic pathways in rheumatic diseases," Nature Reviews Rheumatology, vol. 12, no. 9, pp. 543-558, 2016.

[44] M. Vomero, C. Barbati, T. Colasanti et al., "Autophagy and rheumatoid arthritis: current knowledges and future perspectives," Frontiers in Immunology, vol. 9, p. 1577, 2018.

[45] L. J. O'Neil and M. J. Kaplan, "Neutrophils in rheumatoid arthritis: breaking immune tolerance and fueling disease," Trends in Molecular Medicine, vol. 25, no. 3, pp. 215-227, 2019.

[46] S. Vermeren, K. Miles, J. Y. Chu, D. Salter, R. Zamoyska, and M. Gray, "PTPN22 is a critical regulator of Fc $\gamma$ receptormediated neutrophil activation," Journal of Immunology, vol. 197, no. 12, pp. 4771-4779, 2016.

[47] B. Bartok and G. S. Firestein, "Fibroblast-like synoviocytes: key effector cells in rheumatoid arthritis," Immunological Reviews, vol. 233, no. 1, pp. 233-255, 2010.

[48] C. Sanchez, A. C. Bay-Jensen, T. Pap et al., "Chondrocyte secretome: a source of novel insights and exploratory biomarkers of osteoarthritis," Osteoarthritis and Cartilage, vol. 25, no. 8, pp. 1199-1209, 2017.

[49] I. B. McInnes, C. D. Buckley, and J. D. Isaacs, "Cytokines in rheumatoid arthritis - shaping the immunological landscape," Nature Reviews Rheumatology, vol. 12, no. 1, pp. 63-68, 2016.

[50] A. E. Koch, "Chemokines and their receptors in rheumatoid arthritis: future targets?," Arthritis and Rheumatism, vol. 52, no. 3, pp. 710-721, 2005.

[51] K. S. Nandakumar and R. Holmdahl, "Arthritis induced with cartilage-specific antibodiesis IL-4-dependent," European Journal of Immunology, vol. 36, no. 6, pp. 1608$1618,2006$.

[52] Z. Han, D. L. Boyle, L. Chang et al., "c-Jun N-terminal kinase is required for metalloproteinase expression and joint destruction in inflammatory arthritis," The Journal of Clinical Investigation, vol. 108, no. 1, pp. 73-81, 2001.

[53] N. Raje, E. Terpos, W. Willenbacher et al., "Denosumab versus zoledronic acid in bone disease treatment of newly diagnosed multiple myeloma: an international, doubleblind, double-dummy, randomised, controlled, phase 3 study," The Lancet Oncology, vol. 19, no. 3, pp. 370-381, 2018.

[54] Y. Ikebuchi, S. Aoki, M. Honma et al., "Coupling of bone resorption and formation by RANKL reverse signalling," Nature, vol. 561, no. 7722, pp. 195-200, 2018.
[55] H. D. Jang, H. Z. Hwang, H. S. Kim, and S. Y. Lee, "CCbl negatively regulates TRAF6-mediated NF- $\kappa$ B activation by promoting K48-linked polyubiquitination of TRAF6," Cellular \& Molecular Biology Letters, vol. 24, no. 1, p. 29, 2019.

[56] L. Chang and M. Karin, "Mammalian MAP kinase signalling cascades," Nature, vol. 410, no. 6824, pp. 37-40, 2001.

[57] C. Zhou, Y. You, W. Shen et al., "Deficiency of sorting nexin 10 prevents bone erosion in collagen-induced mouse arthritis through promoting NFATc1 degradation," Annals of the Rheumatic Diseases, vol. 75, no. 6, pp. 1211-1218, 2016.

[58] J. Kikuta and M. Ishii, "Osteoclast migration, differentiation and function: novel therapeutic targets for rheumatic diseases," Rheumatology, vol. 52, no. 2, pp. 226-234, 2013.

[59] A. I. Catrina, C. I. Svensson, V. Malmstrom, G. Schett, and L. Klareskog, "Mechanisms leading from systemic autoimmunity to joint-specific disease in rheumatoid arthritis," Nature Reviews Rheumatology, vol. 13, no. 2, pp. 79-86, 2017.

[60] J. S. Smolen, D. Aletaha, A. Barton et al., "Rheumatoid arthritis," Nature Reviews Disease Primers, vol. 4, no. 1, p. 18001, 2018.

[61] D. O.'. Gradaigh, D. Ireland, S. Bord, and J. E. Compston, "Joint erosion in rheumatoid arthritis: interactions between tumour necrosis factor, interleukin 1, and receptor activator of nuclear factor B ligand (RANKL) regulate osteoclasts," Annals of the Rheumatic Diseases, vol. 63, no. 4, pp. 354-359, 2004.

[62] I. B. McInnes, B. P. Leung, R. D. Sturrock, M. Field, and F. Y. Liew, "Interleukin-15 mediates T cell-dependent regulation of tumor necrosis factor-alpha production in rheumatoid arthritis," Nature Medicine, vol. 3, no. 2, pp. 189-195, 1997.

[63] S. Y. Hwang, J. Y. Kim, K. W. Kim et al., "IL-17 induces production of IL- 6 and IL-8 in rheumatoid arthritis synovial fibroblasts via NF-kappaB- and PI3-kinase/Akt-dependent pathways," Arthritis Research \& Therapy, vol. 6, no. 2, pp. R120-R128, 2004.

[64] H. Takayanagi, S. Kim, K. Matsuo et al., "RANKL maintains bone homeostasis through c-Fos-dependent induction of interferon-beta," Nature, vol. 416, no. 6882, pp. 744-749, 2002.

[65] A. Krabben, A. G. Wilson, D. P. de Rooy et al., "Association of genetic variants in the IL4 and IL4R genes with the severity of joint damage in rheumatoid arthritis: a study in seven cohorts," Arthritis and Rheumatism, vol. 65, no. 12, pp. 3051-3057, 2013.

[66] S. M. Eck, J. S. Blackburn, A. C. Schmucker, P. S. Burrage, and C. E. Brinckerhoff, "Matrix metalloproteinase and G protein coupled receptors: co-conspirators in the pathogenesis of autoimmune disease and cancer," Journal of Autoimmunity, vol. 33, no. 3-4, pp. 214-221, 2009.

[67] P. S. Burrage, K. S. Mix, and C. E. Brinckerhoff, "Matrix metalloproteinases: role in arthritis," Frontiers in Bioscience, vol. 11, pp. 529-543, 2006.

[68] E. E. Mehana, A. F. Khafaga, and S. S. El-Blehi, "The role of matrix metalloproteinases in osteoarthritis pathogenesis: an updated review," Life Sciences, vol. 234, article 116786, 2019. 
[69] Z. Chen, H. Wang, Y. Xia, F. Yan, and Y. Lu, "Therapeutic potential of mesenchymal cell-derived miRNA-150-5pExpressing exosomes in rheumatoid arthritis mediated by the modulation of MMP14 and VEGF," Journal of Immunology, vol. 201, no. 8, pp. 2472-2482, 2018.

[70] S. Zucker, D. Pei, J. Cao, and C. Lopez-Otin, "Membrane typematrix metalloproteinases (MT-MMP)," Current Topics in Developmental Biology, vol. 54, pp. 1-74, 2003.

[71] E. A. Lin and C. J. Liu, "The role of ADAMTSs in arthritis," Protein \& Cell, vol. 1, no. 1, pp. 33-47, 2010.

[72] C. J. Liu, "The role of ADAMTS-7 and ADAMTS-12 in the pathogenesis of arthritis," Nature Clinical Practice Rheumatology, vol. 5, no. 1, pp. 38-45, 2009. 